\title{
Improving Energy Efficiency and Minimizing Backhaul Traffic in LTE-A Network with Cooperation and DTX mechanism
}

\author{
P. Bhuvaneswari, L. Nithyanandan
}

\begin{abstract}
The promising approach to enhance energy efficiency $(E E)$ in LTE-A network is to switch underutilized evolved node $B(e N B)$ to sleep mode, but it increases the transmission power to guarantee the coverage under the remaining active eNBs. The increase in transmission power can be reduced by coordinated multi-point (CoMP) technique through eNB cooperation, but this technique consumes extra power due to backhaul traffic and signal processing. This problem can be solved by the proposed energy efficient acceleration factor failure rate $(E E A F R)$ algorithm. The algorithm jointly considers the eNB cooperation and DTX mechanism to minimize the backhaul traffic with less failure rate. Also, to enhance energy efficiency, the proposed algorithm efficiently utilizes the cooperation in all traffic load scenarios based on the decision criteria and DTX mechanism. The results are compared with the existing AFEE and Green CoMP with Backhaul Traffic algorithm. In all the traffic loads EEAFR save 59.8\% energy and reduces the network outage which depends on the failure rate of the eNB components.

Index Terms--- Backhaul Traffic, Cooperation, DTX, Failure Rate.
\end{abstract}

\section{INTRODUCTION}

The usage of the wireless devices increases day by day in today's world which results in high traffic growth and energy consumption. By 2020 information and communication technology (ICT) is expected to consume higher energy than the production in the overall world. Therefore, the majority of the industries and researchers are focusing on reducing energy consumption in cellular networks. Generally, the network traffic is not constant at all time.

Even the base stations (BS) are working at full power state, the network traffic changes time by time and more energy is utilized in the access network. Hence, to imbalance, the energy consumption between traffic loads by an access network is to put the BS in sleep mode.

To conserve energy in BS, switching ON/OFF strategy play a significant role in cellular network and it faces several challenges.

According to the traffic loads the dynamic ON/OFF switching strategy is investigated in [1] where the network coverage is not affected as shown in Fig. 1 (a). To reduce the consumption of energy in a cellular network, BS is switched off in a low traffic load; then the neighborhood BS need to enlarge the coverage area of the cell to avoid the coverage hole as shown in Fig. 1 ((b) and (c)). The author in [2] proposed CoMP-DESM to solve the coverage problem and improved EE by joint processing cell selection method and

Manuscript received September 16, 2019.

P.Bhuvaneswari, Department of Electronics and Communication Engineering, Pondicherry Engineering College, Puducherry, India.

E-mail: bhuvana.ece06@pec.edu

L. Nithyanandan,Department of Electronics and Communication Engineering, Pondicherry Engineering College, Puducherry, India. without increasing the transmission power the network performance is increased for the edge users.

One of the serious issues in a heterogeneous network is user association problem. The unequal cell sizes in uniform user distribution result uneven traffic loads which are investigated in [3] to select serving BS based on EE and load balancing.

The energy saving algorithm is addressed [4] to reduce the total cost in a dense heterogeneous network for downlink transmission and also an optimal association mechanism for user association problem is proposed for re-association users and new arrival users based on delay and energy tradeoff. Author in [5] investigated the efficient power conservation scheme for LTE-A networks and IEEE 802.16m to optimize the battery life for various traffic patterns. The user scheduling with limited backhaul capacity in a heterogeneous system is studied [6] to perform the BS independently and locally from another BS with capacity limitations and the complexity of scheduling due to the limiting factor of the link between the macro BS and core network. Soft mode and hard mode switching methods are proposed [7] in downlink BS cooperative to improve the sum rate and to reduce the complexity by joint processing CoMP and coordinated beam forming CoMP with limited backhaul.

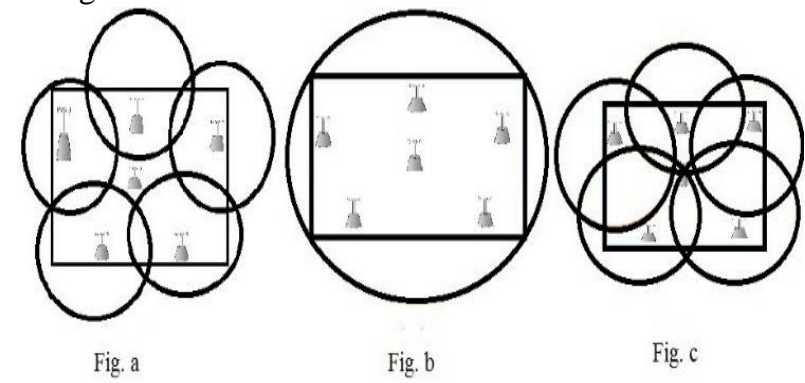

Fig. 1: (a) BS switch off when a coverage hole present (b) BS with high power level (c) BS with a low power level

In a two-tier heterogeneous network, the BS association mechanism [8] reduced the packet delay and improved the performance of the network by backhaul aware scheme with BS association than the traditional backhaul aware method. The author in [9] proposed the two-stage resource allocation algorithm to improve energy efficiency (EE) in heterogeneous networks.

In the first stage, an auction-based power control, joint relay selection and spectrum allocation algorithm are used to maximize EE for D2D to V2V in heterogeneous networks. In the second stage, to improve EE the power control algorithm is used in a nonlinear fractional method.
Blue Eyes Intelligence Engineering $\&$ Sciences Publication 


\section{IMPROVING ENERGY EFFICIENCY AND MINIMIZING BACKHAUL TRAFFIC IN LTE-A NETWORK WITH COOPERATION AND DTX MECHANISM}

The system performance is improved [10] by deterministic approach method through time optimization technique, resource allocation, weighting factors and MUEs in two-tier heterogeneous networks where the network was appropriately used for both downlink and uplink traffic with the weighting factors and the results showed the effect of distance between BS and access points, also the number of MUEs increased when more bandwidth is used. In [11] the EE of the two-tier heterogeneous system is not stable to the network traffic under spatial multiplexing, which can be solved by constraining the number of UEs connected to every single BS.

Hence, following to the traffic condition, the authors [11] designed the bandwidth division among wireless backhaul and radio access links and proved two-tier MIMO heterogeneous network is more efficient when compared to single tier system in terms of energy. To enhance EE in dense small cell networks, CSMPA method is proposed [12] to control the system performance and support the target rates of users. Hence, the number of outage users is very less and satisfied the requirements of the user. The author in [13] introduced the novel framework to reduce power consumption in LTE-A network under low utilized condition. For end users, EEC algorithm is proposed to balance QoS requirements and power consumption with various traffic types. To maximize the fairness among the users and to manage the load balancing in clustered HetNet [14] in LTE-A system, central and distributed SON algorithm are proposed to handle the macroscopic users and CoMP selection for location of the users.

These algorithms reduce ICI and improved the inner small cell base station users and effectively handled the load balancing SON networks. In a double layer HetNet with massive MIMO, dynamic micro cell clustering strategy is investigated for interference coordination [15] and interference can be reduced by graph-based technique to improve the small cell users downlink sum rate with the power constraints of every small cell BS. Author in [16] investigates the problem of UEs cooperation, frame design and resource allocation with wireless backhaul in MIMO HetNet to achieve the downlink sum rate for the users. In [17] AFEE algorithm is proposed to reduce the failure rate when the BS components are put into a DTX state.

Thus, energy efficiency is improved in LTE-A networks. In DTX mechanism, the time taken from one state to another state is $35 \mu \mathrm{s}$. Thus, it reduces energy consumption. In [18] various algorithms with backhaul traffic are addressed to improve energy efficiency with base station cooperation techniques and identified less cooperative size is preferable for throughput enhancement. By combining the issues [17-18], this paper discusses multiple objectives to enhance EE in LTE-A networks by EEAFR algorithm. To this end the contribution of the work are as follows:

i. Initially, the base station cooperation with backhaul technique is considered, which is significantly flexible to manage the user association and to satisfy the UEs requirements depending on the traffic load conditions.

ii. Secondly, DTX mechanism is applied when the cooperative set significantly depends on the serving

area of eNB. Thus, DTX mechanism reduces power consumption in eNB components.

iii. The EEAFR algorithm reduces the Failure Rate of the eNB components when the eNBs are in DTX state and to minimize the backhaul traffic to enhance the EE of the LTE-A networks.

\section{System Model}

\section{A. Architecture}

LTE-A system consists of various kinds of eNBs to manage different user equipment (UEs). As shown in Fig. 2 the system model comprises five macro cells and six microcells where eNBs are surrounded by cells which are represented by numbers and the UE are assigned among eNBs.

The macro eNBs are associated with the core network by fiber link and micro eNBs are connected through wireless backhaul links and the system parameters are listed in Table 1. Each macro eNBs have three-dimensional antennas. Based on the traffic conditions the eNBs cooperate with each other and depends on the quality of service, the transmission rates of UEs are guaranteed and varied in a daytime.

The density of UEs and the eNB traffic load are more in an urban environment at daytime and the traffic load decreases at office hours based on the usage of the mobile phones. Thus, the energy saving can be achieved in an LTE-A network, when the idle eNBs are switched off.

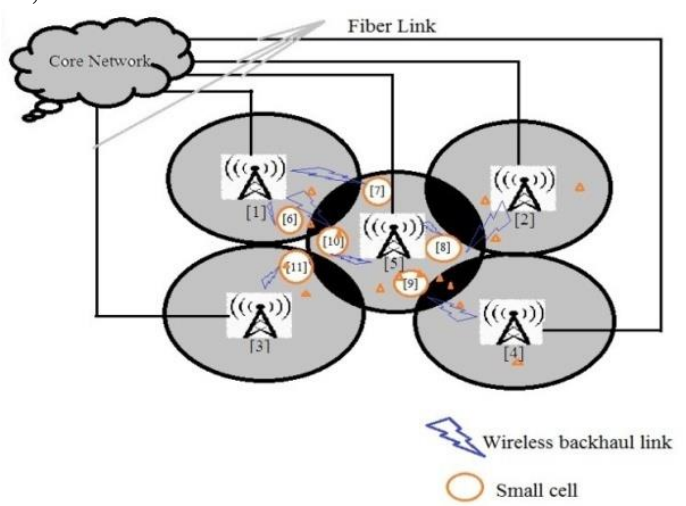

Fig. 2: System Model

As shown in Fig. 3 (a) five macro and micro eNBs are cooperating with other. In this, the possible combination of cooperation is tried such as four eNBs cooperating together one eNB working alone, three eNBs are cooperating together two eNBs working alone. The cooperation is formed when two eNBs are at a determined distance. The algorithm decides which eNB can be put into DTX mode and which eNB formed into a cluster.

Hence, this technique improves energy efficiency in a network. Base station cooperation and DTX mechanism are shown in Fig. 3 and system description is shown in Table 2, to satisfy the user requirements and to enhance the EE.In Fig. 3 (b) macro eNB five and other micro eNBs are working in DTX state to gradually minimize the energy consumption in the system wherein Fig. 3 (c) shows the cell cooperation and UE association. 
Table 1:System Parameters

\begin{tabular}{|l|l|}
\hline \multicolumn{1}{|c|}{ System Parameters } & Value \\
\hline Operating Frequency of eNB & $2 \mathrm{GHz}$ \\
\hline Transmission range for Macro eNBs & $1000 \mathrm{~m}$ \\
\hline Number of RB/eNB for micro and macro & $25 \mathrm{and} \mathrm{50}$ \\
\hline Minimum receiver sensitivity & $-107.5 \mathrm{dBm}$ \\
\hline Cell scaling range & $1500 \mathrm{~m}$ \\
\hline Receiver node power & $-97.5 \mathrm{dBm}$ \\
\hline eNB cooperation for channel gain & $5 \mathrm{~dB}$ \\
\hline $\begin{array}{l}\text { Maximum eNB TX power and antenna gain } \\
\text { for macro }\end{array}$ & $46 \mathrm{dBm}, 16$ \\
\hline $\begin{array}{l}\text { Maximum eNB TX power and antenna gain } \\
\text { for micro }\end{array}$ & $37 \mathrm{dBm}, 16$ \\
\hline
\end{tabular}

Macro eNB three and micro eNBs six and eNB seven cooperate to associate UEs from macro eNB one or eNB five. Therefore, macro eNB one and eNB five are enabled to minimize the coverage with the reduction of power. With cooperative set macro eNB, two can be put into DTX mode.

Moreover, in this work, eNBs are not totally powered off when eNBs are switched to DTX mode and eNBs are still able to broadcast a standard message. Initially, the cooperative set is formed by the iterative pattern. First, eNB is turned on to cover entire UEs under the coverage area.

Then the next eNB is chosen based on the maximum cooperation distance from the first eNB to cover the UEs under the service area. Again the third eNB is chosen from the first eNB with the maximum distance of cooperation. Hence, the third eNB only covers half of the users within the coverage area. This kind of eNB cooperation approach helps to improve energy efficiency network and spectrum efficiency for every UEs. Based on the cooperation and limitation of bandwidth, the current set of eNB is decided to cooperate with one or more eNBs or not. In this work, turning ON less eNBs is considerable to achieve energy efficiency. The heavily loaded eNBs are kept in active state and it can cooperate with the neighboring eNBs for saving energy, thenUE cooperation, UE's notification and state examination are executed to calculate which UEs have to serve to these eNBs.

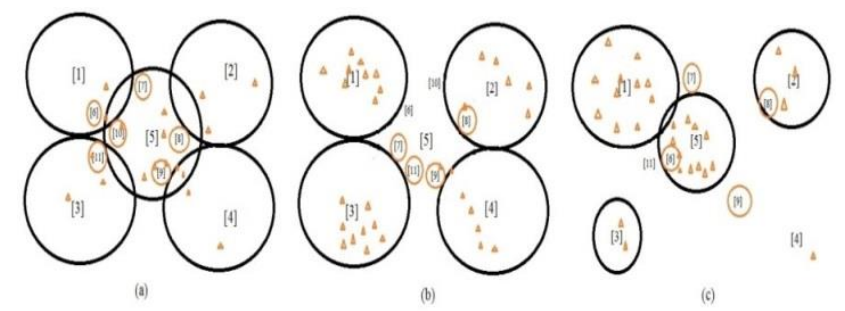

Fig. 3: Cooperating cells with DTX mechanism are denoted as MA, set of micro eNBs are indicated as MI. The binary decision variables are denoted as AC, CXY, PXPQ, SRQ. The available cooperative set AC represents C, where $\mathrm{AC}$ is 1 , indicates the cooperative set is chosen; otherwise $\mathrm{AC}$ is 0 . CXY means UEs $\mathrm{X}$ are connected to cooperative set which represents $\mathrm{Y}$ where CXYis 1 when the UEs are connected by the cooperative set of eNBs Y otherwise CXY is 0 .

The power level (PXPQ) $\mathrm{P}$ is chosen when UEs $\mathrm{X}$ is connected to the cooperative set Q;PXPQ is 1indicatesUEs X formed to the cooperative set $\mathrm{Q}$ by the power level $\mathrm{P}$, otherwise PXPQ is 0.SRQ is1denotes eNB R in cooperative set $\mathrm{Q}$ is chosen and vice versa. The network coverage is
Let the pair of users denoted as $U$, the set of macro eNBs

indicated as B with the binary element of $\mathrm{bXQ}$. The network coverage $b X Q$ is 1 when UEs $X$ is formed into the coverage of cooperative set; otherwise, bXQ is 0 . In the proposed work, the power control, $\mathrm{B}$ and the binary element bXQ is expressed as

$$
\begin{aligned}
& B=\left[\begin{array}{ccc}
b 11 & b 21 \cdots & b M 1 \\
\vdots & \ddots & \vdots \\
b 1 U & \cdots & b M U
\end{array}\right] \\
& B_{X Q}=\left\{\begin{array}{c}
1, \text { if } \sum_{P=Y_{Q X}}^{P c} P_{X P Q}=1, \forall Y \in U, \forall \quad Y \in S \\
0, \text { otherwise }
\end{array}\right.
\end{aligned}
$$

where $\sum_{\mathrm{P}=\mathrm{Y}_{\mathrm{QX}}}^{\mathrm{Pc}} \mathrm{S}_{\mathrm{XRQ}}$ is the total power control PXPQfromthe exact power level YQX to the maximalpower level PC when the UE X is formed to the cooperative set Q. The UEs $X$ are still connected by the cooperative set $Q$ when the power level exceedsYQX. The rate demand ZXQ of UEs $\mathrm{X}$ in cooperative set $\mathrm{Q}$ dependson UEs and spectral efficiency. The capacity limit of UEs in the cooperative set is denoted as $\mathrm{Z}_{\mathrm{Q}}^{\max }$ and the minimum power control is expressed as $\min$

$$
\begin{aligned}
& P_{C T o t}=\left\{\sum_{Q \in C} A_{C}\left(P_{C(Y, Q)}+\eta^{-1} P_{C(M, Q)}\right)+\right. \\
& \left.\sum_{Q \in C} \sum_{X \in Y} C_{X Y} P_{C(K, Q)}\right\}
\end{aligned}
$$

where $P_{C(Y, Q)}, P_{C(M, Q)}, P_{C(K, Q)}$ are the energy consumption variables which is formed by the cooperative set $Q$.

$$
\sum_{Q \in C} C_{X Y}=1, \forall X \in U
$$

The equation (4) expressed each UEs must be connected to an individual cooperation and the overall insistence of the connected UEs would not outperform the bandwidth limit of the individual cooperation expressed as

$$
\begin{aligned}
& \sum_{X \in U} C_{X Y} Z_{X Q} \leq Z_{Q}^{\max } A_{C}, \forall Y \in C \\
& C_{X Y} \leq B_{X Q}, \forall Y \in C, \forall X \in U \\
& \quad \text { if } A_{C}=1 \text {, then } \sum_{k \in C_{K}} L_{K C=}\left|C_{K}\right| \\
& \sum_{Q \in C_{K}} L_{K C} \leq 1, \forall K \in C
\end{aligned}
$$

where $A_{C}, C_{X Y}, L_{K C}$ and $P_{X P Q}$ belongs to $\{0,1\}$. (6) indicates, UEs are formed into the cooperative set when UEs are within the coverage area. (7) Indicates that the cooperative set Q is chosen, all the UEs $K$ should be equal to 1 in the cooperative set and each eNBs in one cooperative set which is shown in equation (8).

Due to more number of UEs in daytime and large set of cooperation $Q$ is considered to cover the number of UEs, the eNB components are overheating which leads to failure rate (FR) and increases the power consumption of the network. This can be solved by introducing a DTX mechanism. This mechanism activates/deactivate some of the eNB components to enhance the network EE in LTE-A systems. DTX is applied to the eNBs when the radiated power $\left(R_{P C}\right)$ of all eNBs is 0 , expressed as

$$
R_{P C} \leq H_{X L t} \forall \in L, t
$$

where $H$ is a constant variable. The operating temperature of eNB components is reduced when DTX mechanism is applied. The change in operating temperature $(T)$ of eNB components $\left(\mu^{T}\right)$ with the reduction of failure rate $\left(\mu^{0}\right)$ can be expressed as

$$
\mu^{T}=\mu^{0} e^{-\frac{E a}{k T}}
$$

where $E_{a}$ is the operating energy and $k$ is the Boltzmann constant. 


\section{IMPROVING ENERGY EFFICIENCY AND MINIMIZING BACKHAUL TRAFFIC IN LTE-A NETWORK WITH COOPERATION AND DTX MECHANISM}

Generally, the impact of failure rate has either positive or negative based on acceleration factor. If $\mathrm{Af}<1$, the impact of failure rate is positive or else the impact of failure rate is negative $(\mathrm{AF}>1)$. The temperature of eNB components are changed in power state transition and the effect of failure rate $\left(\mu^{t r}\right)$ is expressed as thermal cycling frequency and failure cycles are given by

$$
\mu^{T}=\frac{\check{\text { Afft }}}{f}
$$

Table 2: System Description

\begin{tabular}{|l|l|}
\hline Symbol & Description \\
\hline$M_{A}$ & Set of macro eNBs \\
\hline$M_{I}$ & Set of micro eNBs \\
\hline$M$ & Set of eNBs \\
\hline$U$ & Set of UEs \\
\hline$C$ & Cooperative set \\
\hline$P_{c}$ & Set of Power control \\
\hline$A_{C}, C_{X Y}, P_{X P Q}, S_{R Q}$ & Decision binary variables \\
\hline
\end{tabular}

B. Backhaul Topology

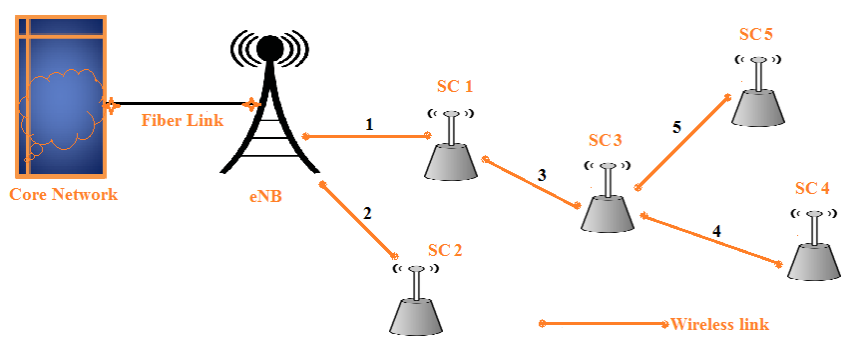

Fig. 4: Backhaul topology

eNB is connected to the core network via the aggregation gateway through a definite number of $\mathrm{BH}$ links which form the network called backhaul. The connection between the access network to core network can be either star topology (direct connection) or tree topology (by many numbers of SC gateways). As shown in Fig. 4 the fiber link connects the core network to eNB and the SC is connected to eNB through the wireless $\mathrm{BH}$ links which are denoted as $W_{B H}$. There are 5 macro eNBs and number of micro eNBs with limited resource blocks. The routing path is denoted as $W_{B H}(R)$, the set of all BH links $S \in W_{B H}$ along with the BH links and routing path, the traffic is directed to an aggregation point from eNB $R$. It can be depicted very clearly in Fig. 4 i.e., the routing path of 1 and 3 is noted as $W_{B H}(1)=\{1\}$ and $W_{B H}(5)$ $=\{1,3,5\}$. The set of all eNB is denoted as $W_{B H}(S)$, where $R$ $\epsilon W$, where traffic is routed over BH link $S$. i.e., $W(1)=\{1,3$, $5,4\}$ and $W_{B H}(3)=\{3,5,4\}$. The BH link $R \in W_{B H}$ is characterized by downlink capacity. The backhaul traffic is minimized when eNB cooperation satisfies the UEs requirements. Let $M$ denotes the set of macro and micro eNBs and coverage area of all eNB denoted as $D$. At location $i$ the mobile UEs associated with eNB $a$. According to M/M/1 queue, the traffic occurs ate $\mathrm{NB} a$ 's backhaul toward the UEs with the arrival rate. The UEs at $i$ the traffic demand and the average required service time in eNB $a$ 's backhaul is

$$
\bigoplus(i)=\frac{u(i)}{E_{a}}
$$

The UEs generated the average traffic at a location $i$ in eNBs $a$ 's backhaul is

$$
\Psi_{a}(i)=\frac{D(\mathrm{i}) u(i) \eta_{a}(i)}{E_{a}}
$$

where $\eta_{a}(i)$ is the indicator function which is represented as $\{0,1\}$. If the indicator function is 1 then UEs at $i$ is associated with eNB $a$ otherwise, UEs are not associated. At location $i$ the average traffic load in eNB $a$ 'backhaul when the mobile UEs are distributed uniformly in the coverage is expressed as

$$
\begin{aligned}
& \rho_{a}=\int_{a \in \overline{\mathrm{G}}} \Psi_{a}(i) d x \\
& K_{a}(i)=\frac{u(i)}{E_{a}\left(1-\rho_{a}\right)} \\
& T_{a}(i)=W_{a}(i)-\Psi_{a}(i) \\
& \Phi_{a}(i)=\frac{T_{a}(i)}{\rho_{a}(i)}=\frac{\rho_{a}}{\left(1-\rho_{a}\right)} \\
& \Phi_{a}\left(\rho_{a}\right)=\frac{\rho_{a}}{\left(1-\rho_{a}\right)}
\end{aligned}
$$

where $K_{a}(i)$ is the average traffic delivery time at location $i$ in eNBs $a$ 's backhaul, $T_{a}(i)$ is the average waiting time in eNB's backhaul $a$. The latency ratio is denoted as $\Phi_{a}(i)$ which measures how much UEs at location $i$ is waiting for service in eNBs $a$ backhaul. The smaller $\Phi_{a}\left(\rho_{a}\right)$ indicates less backhaul traffic in a network to the associated users.

\section{Proposed Algorithm - EEAFR}

The main objective of the work is to enhance energy efficiency in LTE-A network by cooperating eNBs and reducing the failure rate by introducing DTX mechanism when the eNB cooperates large cooperative set. Initially, the proposed algorithm selects the eNB with a heavy load to powered ON [18] and calculate the size of the cell. Then, based on the load, the algorithm decides which eNB to cooperate. Then UE cooperation, UEs notification and state examination are checked to calculate which UEs connected to these eNBs and network information is updated after performing the lightly and heavily loaded eNBs as shown in Fig. 5

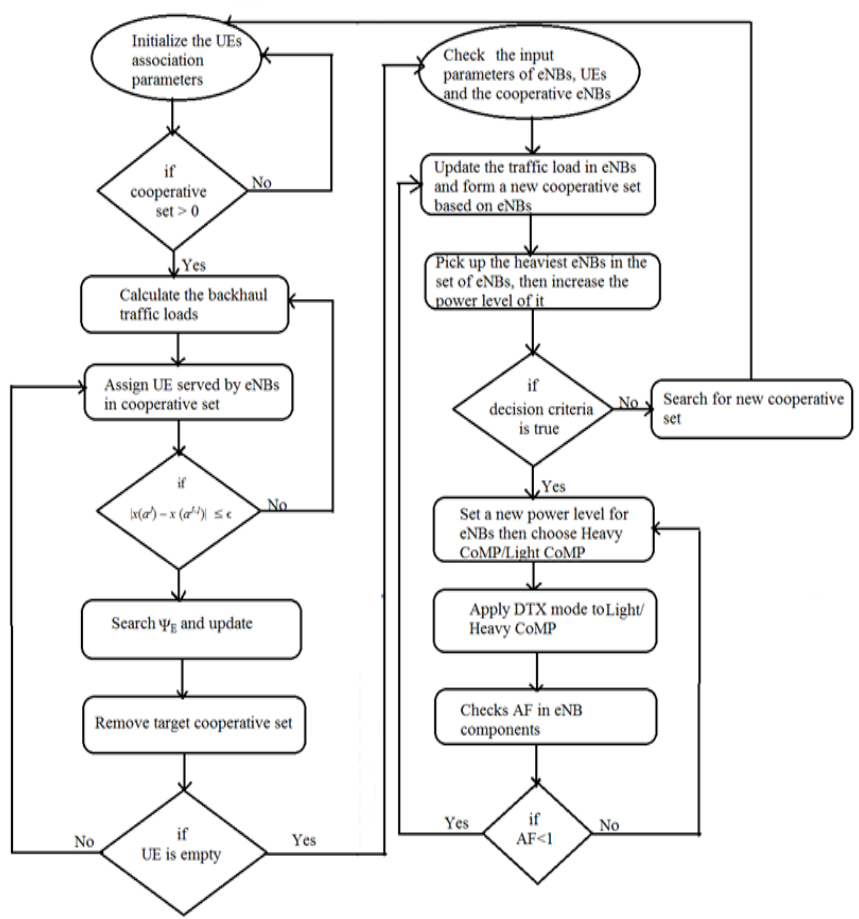

Fig. 5: Proposed Algorithm

The algorithm selects UEs from the nearest eNBs and deletes it from the set of UEs then it calculates UEs under cooperative set. 
For cooperation, the eNBs should need to enlarge the service area and the power level to use based on UE association. The benefit of increasing the power level is to increase the cooperating eNBs. Increasing power level leads to significant power consumption in a network. So that the eNB decides to choose light/heavy loaded eNBs for a cooperative set. The algorithm must decide to choose heavy load eNBs, when there are neighboring eNBs around this heaviest eNBs. Then decision criteria for cooperation technique are examined to enhance the EE of the network to increase the number of the cooperative set for the network improvement. If decision criteria for cooperation technique are not examined, then the algorithm selects next heavy load eNBs among the neighboring eNBs to improve the network performance and hence to increase the energy efficiency in a network. Then the algorithm checks the cooperative set is larger based on original cooperative set after completing the process of decision criteria. In this case, the algorithm chooses a more cooperative set when the cooperative set has not exceeded the original cooperative set. After a larger cooperative set (cooperative exceeded) the algorithms check the neighboring eNBs around the target eNBs. In this case, the algorithm checks the UE association, updating and state examination. Depending on the UE's under cooperating and extended area, the algorithm decides whether to put the eNBs in DTX mode or not. Due to larger cooperation, the eNB devices are overheating hence leads to failure. Average acceleration factor is calculated based on individual eNBs whether the eNB components increase/decrease the failure rate or not. DTX is applied only when the condition $\mathrm{AF}<1$. As a result, the eNB has the initial failure, which can be solved before it attains the acceptable failure rate $(A F>1)$. Finally, the eNBs are arranged in the increasing number of eNBs to check the failure rate of individual eNBs based cooperation. Hence, the total backhaul traffic is less in the proposed algorithm.

\section{Simulation Results}

The performance of AFFE, Green CoMP with $\mathrm{BH}$ and EEAFR algorithm is simulated in an LTE-A network with the performance metrics of energy saving ratio, energy consumption, backhaul traffic, network overhead and network throughput. The proposed algorithm EEAFR is compared with two energy efficient algorithm AFEE and Green CoMP with BH. Green CoMP with BH algorithm reduces energy consumption by eNB cooperation and reduces the backhaul traffic in a network. In AFEE algorithm the energy efficiency is achieved by sleep mode mechanism which reduces the failure in eNB components where the components are overstressed and the usage of mobile devices is high in a daytime. Based on these two algorithms, EEAFR is proposed to improve the LTE-A network as more energy efficient. Finally, the proposed algorithm is compared with the existing algorithms.

The DTX is applied when the eNB has chosen a larger cooperative set to cover UE's under the coverage area. In such a case the eNB components are overheating due to larger cooperation. Hence, failure occurs in the components. As shown in Fig. 6, at $7^{\text {th }}$ eNB, the proposed algorithm has less failure than the existing algorithms because the algorithm accurately decides to put the eNB in DTX mode when larger cooperation occurs.

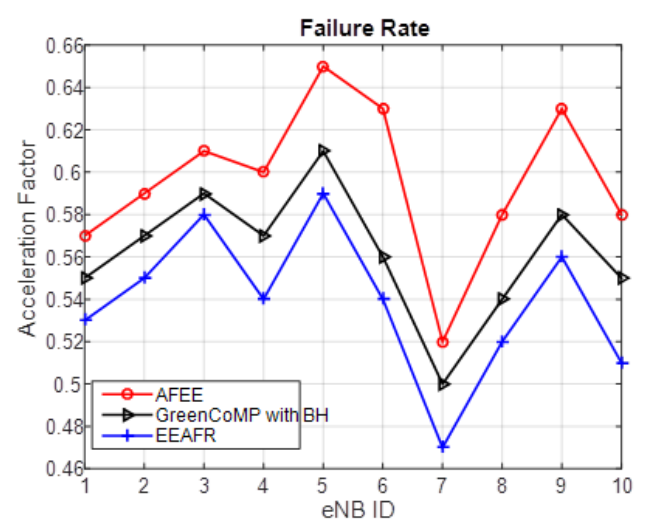

Fig. 6: Failure Rate

The proposed algorithm has less energy consumption than the existing algorithms as shown in Fig. 7. Less cooperation needed for less number of UEs and idle eNBs are to be powered off when UEs are not in coverage area. For more UE's, maximum cooperation is required, which leads to high power consumption. This can be solved by the backhaul traffic with the proposed algorithm and simultaneously manage the eNB components with less failure rate to save $59.8 \%$ energy as shown in Fig. 8. Thus, the network is "green" in all traffic load scenarios and minimum/maximum cooperation. Hence, the proposed algorithm is more efficient than the existing algorithm.

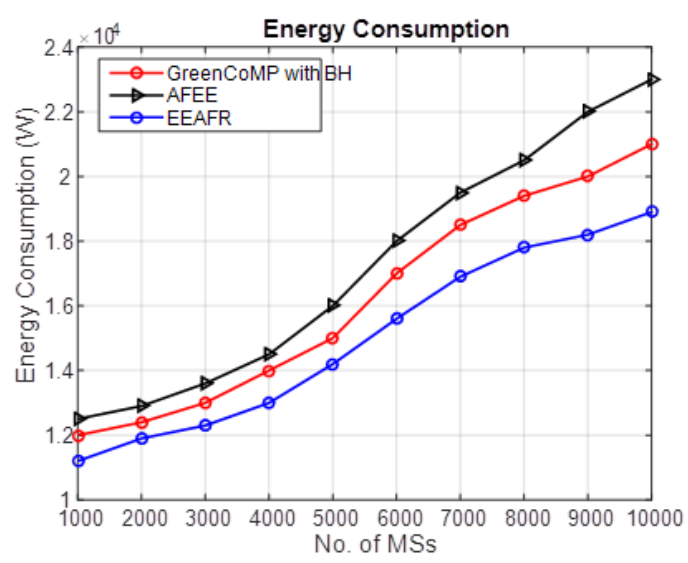

Fig. 7: Energy Consumption

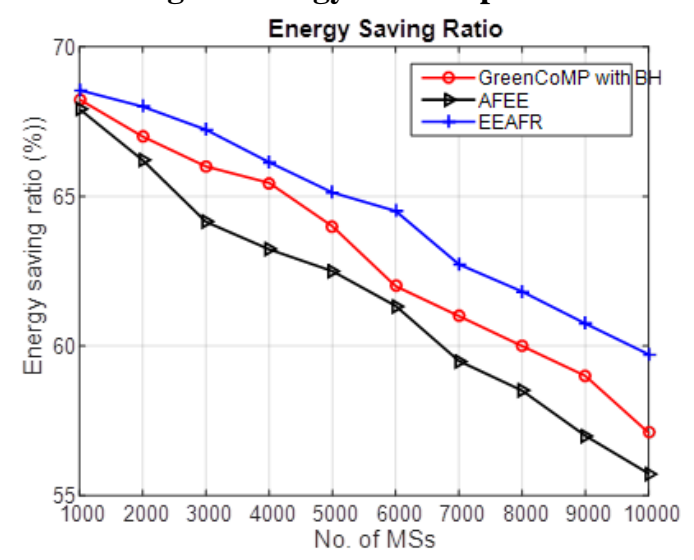

Fig. 8: Energy Saving Ratio 


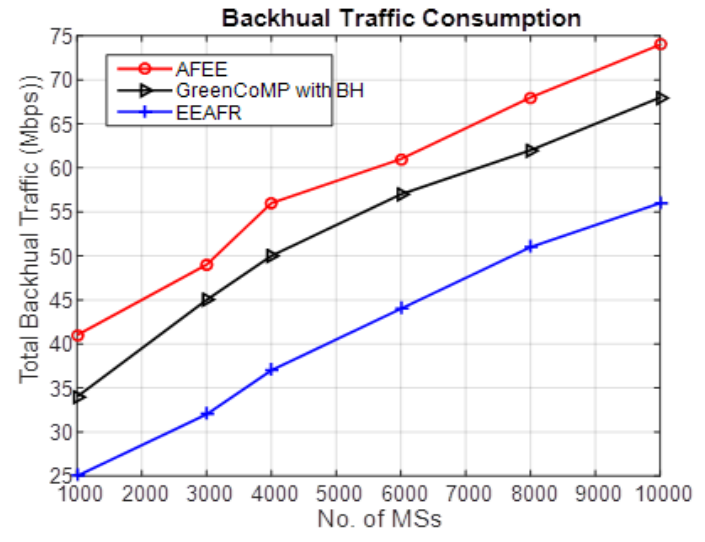

Fig. 9: Backhaul Traffic

Under the proposed and existing algorithms, the impact of backhaul traffic is as shown in Fig. 9. When the MSs increases, the total backhaul consumption also increases. This is due to more UEs requests services such that more eNBs should be cooperated to satisfy the requirements. EEAFR has less traffic consumption than the existing algorithm because the cooperation takes place when the UE's request service to the eNBs while in the existing algorithm the size of the network is smaller. Therefore, the network performance is reduced. Hence the proposed algorithm reduces the backhaul traffic consumption.

The increasing cooperation distance reduces energy consumption as shown in Fig. 10. The higher order cooperation is not preferable in existing algorithm which reduces throughput enhancement in the network which is carefully examined in EEAFR algorithm. EEAFR algorithm out performs the existing algorithms in all cooperation distances by DTX mechanism.

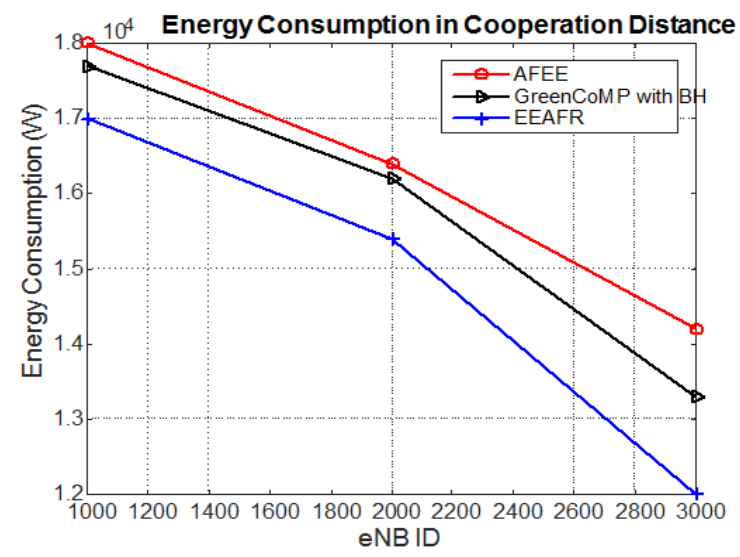

Fig. 10: Energy Consumption for cooperating distance of eNBs

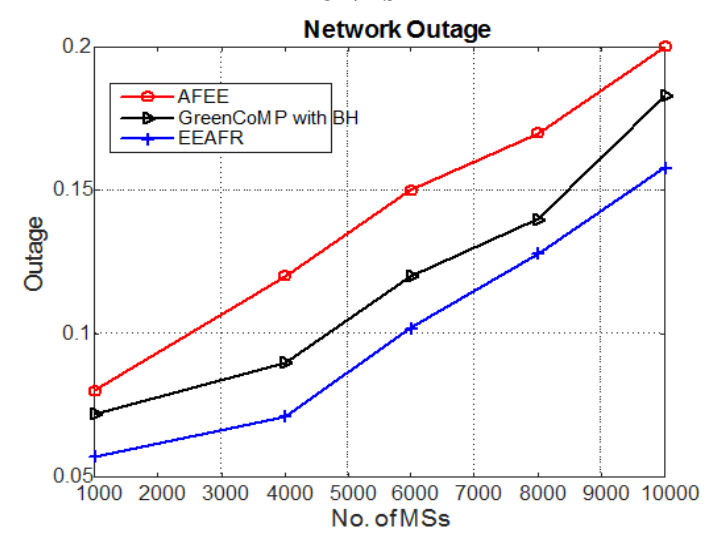

Fig. 11: Network Outage

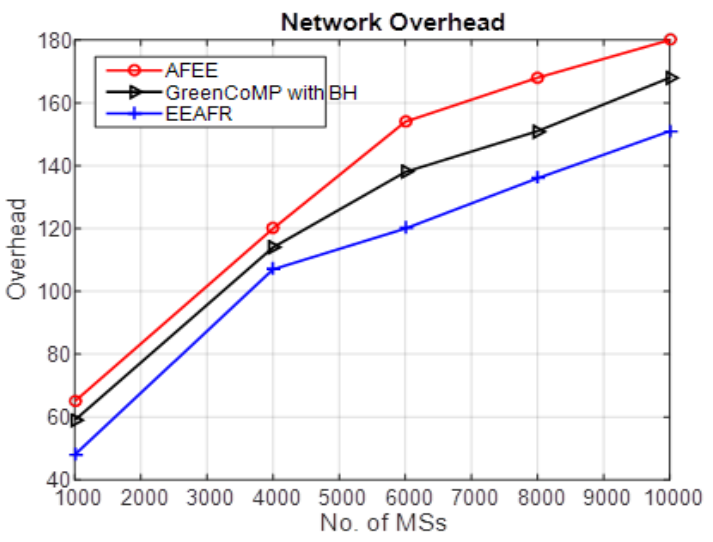

Fig. 12: Network Overhead

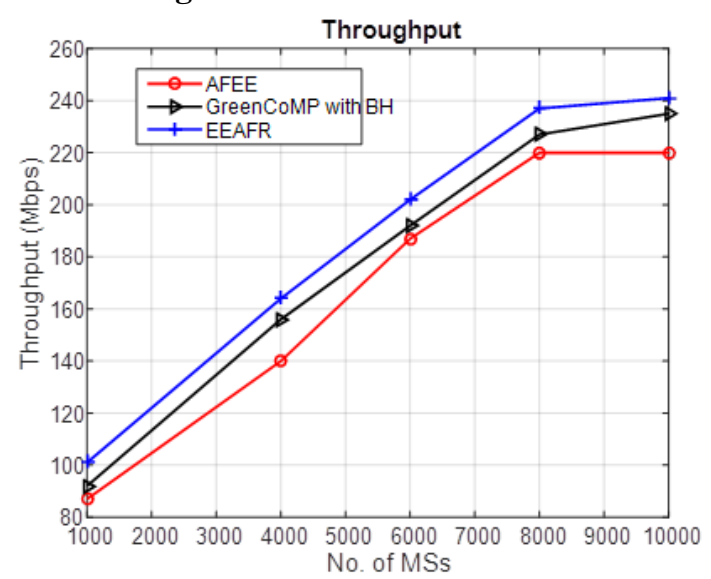

Fig. 13: Throughput

In Fig. 11 the network outage is low in EEAFR algorithm than the existing algorithm because when the cooperation is maximum the DTX is applied to reduce the consumption of power.

The major consumption of power takes place when the eNB components are over stressing which causes failure. The existing algorithms have a stronger outage due to more number of eNBs are activated for high traffic conditions which leads to significant failure in the eNB components. The outage issue can be solved by simultaneously managing the eNB cooperation with backhaul traffic and DTX mechanism for the proposed algorithm.

Network overhead is less for the proposed algorithm as shown in Fig. 12. The reason is, the proposed algorithm effectively satisfying the UE's requirement by cooperating eNBs and powered off eNBs when less UE's request services. Also, the proposed algorithm decides when to apply DTX mode to reduce the overhead and managing the backhaul traffic in entire network. This cannot be achieved in the existing algorithm.

In Fig. 13 the network throughput for the proposed algorithm increases when the network capacity increases to satisfy UE's demand by maximum cooperation. Even there is a large number of UEs request services the proposed algorithms significantly outperform existing by cooperation with DTX mechanism which reduces the failure in eNB components. Therefore, the network performance is increased by EEAFR algorithm.

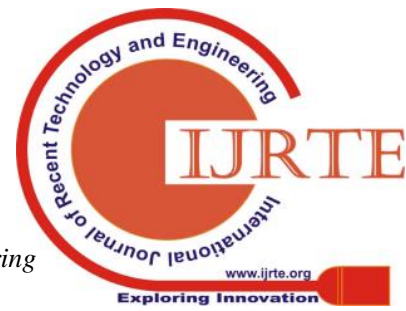




\section{CONCLUSION}

The Cooperation and DTX mechanism is proposed to improve energy efficiency in LTE-A networks and reduces the failure rate in eNB components. The two-energy efficient algorithm is compared (AFFE and Green CoMP with B) with the proposed algorithm - EEAFR which improved network performance and reduces the failure in eNB components when the cooperation eNBs are more enough to satisfy the UEs requirements under coverage area and reduces the backhaul traffic in the LTE-A network. The proposed algorithm effectively utilizes the DTX mechanism in eNB cooperation in all traffic loads. Hence the energy consumption and network outage are reduced. Therefore, LTE-A network saves $59.8 \%$ energy than AFEE and Green CoMPwith $\mathrm{BH}$ algorithm. Thus, the EEAFR algorithm is "Green" enough in LTE-A network.

\section{REFERENCES}

1. E. Oh and B. Krishnamachari, "Toward dynamic energy efficient operation of cellular network infrastructure," IEEE Communications Magazine. 49 (6), pp. 56-61, 2011.

2. Fei Zheng and Wenjing Li, "Distributed Energy Saving Mechanism Based on CoMP in LTE-A System," China Communication on Network Routing and Protocol, 13(7), pp. 39-48, 2016.

3. Y. Chen and J. Li, "User association with unequal user priorities in heterogeneous cellular networks," IEEE Trans. on Vehicular Technology, 65(9),pp. 7374-7388, 2016.

4. Lun Tang and Weili Wang, "An Energy-Saving algorithm with joint user association, clustering and On/Off strategies in dense heterogeneous networks," IEEE Access, 5(17),pp. 12988-13001, 2017.

5. Ronny Yongho Kim and ShantidevMohanty,"Advanced Power Management Techniques in Next-Generation Wireless Networks," IEEE Communications Magazine, 48 (5), pp. 94-102, 2010.

6. Jagadish Ghimire and Catherine Rosenberg, "Revisiting Scheduling in Heterogeneous Networks When the Backhaul is Limited," IEEE Journal on Selected Areas in Communications, 33 (10), pp. 2039-2051, 2015.

7. Qian Zhang and Chenyang Yang, "Downlink Base Station Cooperative Transmission under Limited-Capacity Backhaul," IEEE Trans. on Wireless Communications, 12 (8), pp. 3746-3760, 2013.

8. GongzhengZhangand Tony Q. S. Quek, "Backhaul-aware Base Station Association in Two-tier Heterogeneous Cellular Networks," IEEE $16^{\text {th }}$ International workshop on signal processing advances in wireless communication, pp. 390-395, 2015.

9. Zhenyu Zhou andFeiXiong, "Energy-efficient Vehicular Heterogeneous Networks for Green Cities," IEEE Trans. on Industrial Informatics, 14 (4), pp. 1522-1539, 2018.

10. Wenchao Xia, Jun Zhang and Shi Jin, "Large System Analysis of Resource Allocation in Heterogeneous Networks with Wireless Backhaul," IEEE Trans. on Communications, 65 (11), pp. 5040-5053, 2017.

11. Howard H. Yang, Giovanni Geraci and Tony Q. S. Quek, "Energy-Efficient Design of MIMO Heterogeneous Networks with Wireless Backhaul," IEEE Trans. on Wireless Communications, 15 (7), pp. 4914-4927, 2016.

12. Shie Wu, Zhimin Zeng and Hailun Xia, "Coalition-Based Sleep Mode and Power Allocation for Energy Efficiency in Dense Small Cell Networks," IET Communications, 11 (11), pp. 1662-1670,2017.
13. MugeErelOzcevik, GokhanSecinti and Berk Canberk, "QoS-Aware Power Management in LTE- A Networks under Heterogeneous Traffics," IEEE Trans. on Vehicular Technology, 67 (1), pp. 674-688, 2018.

14. Jin-Bae ParkandKwang Soon Kim, "Load-Balancing Scheme with Small-Cell Cooperation for Clustered Heterogeneous Cellular Networks," IEEE Trans. on Vehicular Technology, 67 (1), pp. 633-649, 2018.

15. Wanming Hao, Osamu Muta and HarisGacanin, "Dynamic Small Cell Clustering and Non-Cooperative Game-Based Precoding Design for Two-Tier Heterogeneous Networks With Massive MIMO," IEEE Trans. on Communications,66 (2),pp. 675-688, 2018.

16. Mingjie Feng, Shiwen Mao and Tao Jiang, "Joint Frame Design, Resource Allocation and User Association for Massive MIMO Heterogeneous Networks with Wireless Backhaul," IEEE Trans. on Wireless Communications, 17 (3), pp. 1937-1950, 2018.

17. P. Bhuvaneswari and L. Nithyanandan, "Improving energy efficiency in LTE-A networks with the reduction of failure rate in eNB components," Wiley Journal of Concurrency and Computation: Practice and Experience.31, pp. 1-10, Oct. 2018.

18. P. Bhuvaneswari and L. Nithyanandan, "Improving energy efficiency in Backhaul of LTE-A Network with Base Station Cooperation," in Proceedings of 8th International Conference on Advances in Computing and Communication (ICACC-2018), Elsevier 143, pp. 151-157, Nov. 2018.

\section{AUTHORS PROFILE}

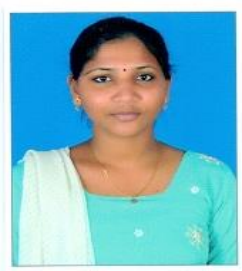

Miss. P. Bhuvaneswari received her B. Tech degree in Electronics and Communication engineering in 2010 from Pondicherry University and $\mathrm{M}$. Tech degree in wireless communication from Pondicherry Engineering College, Pondicherry University in 2012, where she is currently pursuing the Ph.D degree in the department of Electronics and Communication Engineering. Her research interests are in Long Term Evolution - Advanced (LTE-A) networks, to improve energy efficiency in LTE-A networks.

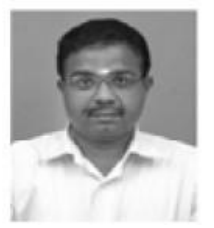

Dr. L. Nithyanandan received his B.E degree in Electronics and Communication in 1992 from the University of Madras, Chennai and M. Tech degree in 1999 from Pondicherry Engineering College. $\mathrm{He}$ received his $\mathrm{Ph} . \mathrm{D}$ degree in wireless communication, 2006, in Pondicherry engineering college, Puducherry. $\mathrm{He}$ is currently a professor in the department of Electronics and Communication engineering, Pondicherry engineering college. His research includes Wireless Communication, Satellite Communication, Antennas, Telemedicine and multi-carrier communication and Next generation wireless networks. He is a Life member of ISTE. 